\title{
Design, Optimization and Performance Analysis of Condenser for HVAC Automobile System for R-290.
}

\author{
Parikshit A. Ladke* and C.S. Choudhari \\ ${ }^{\dagger}$ MechanicalDepartment, Savitribai Phule Pune University, AISSMS, COE, Pune, India
}

Accepted 15 June 2016, Available online 20 June 2016, Special Issue-5 (June 2016)

\begin{abstract}
In automobile air conditioning system condenser plays a vital role. In this paper theoretical and experimental study of acondenser for automobile air conditioning is carried out with refrigerant propane (R290). In automobile air conditioning R134a is widely usedrefrigerant whichis not environmental friendly because of its higher GWP value hence there is need of to replace R134a with the environmentally friendly refrigerant like hydrocarbon (i.e. R290). The optimization of condenser is done by varying different geometric parameters and the performance of the condenser is studied experimentally by carried out the experimentation. In optimization of condenser the optimum value for the condenser is obtained is 5.2 outer diameter, 2 rows and 12 fin per inch and in experimental analysis at 6 $\mathrm{m} / \mathrm{s}-7 \mathrm{~m} / \mathrm{s}$ air velocity gives optimum result are obtained.
\end{abstract}

Keywords:Alternative refrigerants, Condenser, Optimization, R290

\section{Introduction}

The heating, ventilation and air conditioning of the automotive system is designed to provide comfort to driver and passengers. One of the important things to be fulfilled is comfort. Human comfortis the state of mind that expresses satisfaction with the surrounding environment according to American Society of Heating, Refrigeration and Air Conditioning Engineers (ASHRAE).The main function of an air conditioning control system is to modulate the A/C system capacity to match the design condition and climate change, in order to maintain the indoor environment within desirable limits at optimum energy use levels during the entire drive duration. One of the important part in air conditioning system is condenser. The condenser is a heat exchanger located at front of the vehicle and receives high-pressure, hot refrigerant from the compressor. In air conditioning system refrigerant flows through the condenser and cools off from the wind when driving at highway speeds or air blowing from electric cooling fan. A condenser in which desuperheating of high temperature vapor changes the phase from vapour to liquid and sub cooling of condensate occurs. The condenser is an important device and the main function of condenser is to remove heat of hot vapor refrigerant discharged from the compressor. The hot vapor refrigerant contains the heat absorbed by the evaporator and the heat of compression due to the mechanical energy of the

*Corresponding author: Parikshit A. Ladke compressor motor. The heat from the hot vapor refrigerant in a condenser is removed by transmitting it to the walls of the condenser tubes and then from the tubes to the cooling medium. The cooling medium is generally air or water.

J.B. Copettiet. al. have designed and optimized the minichannel parallel flow condenser with refrigerant R-134a for automobile air conditioning system. Optimization was done through variation of geometric parameter like refrigerant side pass arrangement, number of channels, height and width of the tube.

Viveksahuet. al. experimentally studied the wire on tube condenser with different spacing of wire. The optimization was done by varying operating parameter like condenser pressure, condenser temperature.

In present study, optimization of condenser carried out by varying different geometrical parameter and experimental analysis done by varying air velocity through condenser fan.

\section{Propane}

Propane (R290) is an eco-friendlynatural refrigerant.Which has very low Global Warming Potential and zero Ozone Depletion Potential and has no direct impact on the greenhouse effect. It is a Hydrocarbon refrigerant. Propane has excellent thermal performance, low price, and R290 can be compatible with general machinery lubricants and structural material. 


\subsection{Thermophysical Properties}

Molecular weight of weight of R290 is less as compared to other refrigerant. R290 has the slightly highest critical temperature; the highest is the latent heat of vaporization that normally means higher efficiency. Lower saturation pressure allows ease in manufacturing of refrigeration system.

Table 1Thermophysical property

\begin{tabular}{|c|c|}
\hline Refrigerant & R290 \\
\hline Molecular Weight & 44.10 \\
\hline Normal B.P. ${ }^{0} \mathrm{C}$ & -42.2 \\
\hline Critical Temp. Tc ${ }^{0} \mathrm{C}$ & 96.7 \\
\hline Critical Press MPa & 4.25 \\
\hline Latent heat of Evap. kJ/kg & 425.4 \\
\hline
\end{tabular}

\subsection{Environmental characteristics}

Atmospheric life of R-290 is very less as compared to other which means it will sustain in environment for very less time and hence it is environment friendly. HC290 is a non-ozone depleting refrigerant whereas most of the refrigerant is a non-zero ODP refrigerant. GWP value of HC-290 is 20, which is very low compared to other.

Table 2 Environmental characteristics

\begin{tabular}{|c|c|}
\hline Refrigerant & R290 \\
\hline Atm. life (Years) & 0.041 \\
\hline ODP(R11=1) & 0.000 \\
\hline GWP100yr(C02=1) & 20 \\
\hline
\end{tabular}

\subsection{Safety characteristics}

For the development of HC-290, flammability and toxicity are very important parameters due which it was neglected alternative for so many years. R290 is classified under A3 safety class as per ASHRE34-2010 due to this it has been avoided. But it has good thermo physical properties which is similar to R22, hence can be effectively used with taking proper care of leakage factor during operation. The European standard EN378 gives the safety requirements for the use of flammable refrigerants in various applications. Toxicity safe index for R22 and R290 are similar.

Table 3 Safety characteristics

\begin{tabular}{|c|c|}
\hline Refrigerant & R290 \\
\hline LFL by mass $\mathrm{kg} / \mathrm{m}^{3}$ & 0.075 \\
\hline LFL by volume $\%$ & 2.1 \\
\hline Burning velocity $\mathrm{cm} / \mathrm{s}$ & 46 \\
\hline Combustion heat MJ/kg & 50.3 \\
\hline Toxicity ppm & 1000 \\
\hline Safety class & $\mathrm{A} 3$ \\
\hline
\end{tabular}

11| MIT College of Engineering, Pune, India, MECHPGCON 2016, INPRESSCO IJCET Special Issue-5 (June 2016)
When considering a mixture of a flammable fluid and air, burning or explosion can occur upon contact with a source of ignition in the case that the mixture concentration is between the lower explosive limit (LEL) and the upper explosive limit (UEL) and Stored in a cool, ventilated coffers away from fire and the heat source. Propane sensors have been installed nearby the compressor and inside electrical panels. These sensors detect from $0-20 \%$ of the LEL and different safety levels have been considered depending on the concentration measured by the sensors.

\section{Design of condenser}

Design of condenser is done by using various correlations to calculate the heat transfer coefficient from air side and refrigerant side. Heat transfer of finned tube air cooled condenser is given by

$$
Q=U_{t} A_{t} L M T D
$$

$$
L M T D=\frac{\Delta T_{1}-\Delta T_{2}}{\ln \left(\frac{\Delta T_{1}}{\Delta T_{2}}\right)}
$$

$$
U_{t}=\frac{1}{\frac{1}{h_{i}} \frac{A_{t}}{A_{i}}+\frac{1}{h_{f}}+\frac{A_{t}}{A_{i}} \frac{r_{i} \ln \left(d_{o} / d_{i}\right)}{K_{w}}+\frac{1}{h_{o} \eta_{o}}}
$$

\subsection{Air side heat transfer}

Air side heat transfer coefficient calculated using wang and chi correlation (2000).

$$
j=\left\{\begin{array}{ll}
0.108 \operatorname{Re}_{d c}{ }^{-0.29}\left(\frac{X_{t}}{X_{l}}\right)^{c_{1}}\left(\frac{p_{f}}{d_{c}}\right)^{-1.084}\left(\frac{p_{f}}{D_{h}}\right)^{-0.786}\left(\frac{p_{f}}{X_{t}}\right)^{c_{2}} & \text { for } N_{r}=1 \\
0.086 \operatorname{Re}_{d c}^{c_{3}} \cdot N_{r} c_{c_{4}}\left(\frac{p_{f}}{d_{c}}\right)^{c_{5}}\left(\frac{p_{f}}{D_{h}}\right)^{c_{6}}\left(\frac{p_{f}}{X_{t}}\right)^{-0.93} & \text { for } N_{r} \geq 2
\end{array}\right\}
$$

Where

$$
\begin{aligned}
& c_{1}=1.9-0.23 \ln R E_{d c} \\
& c_{2}=-0.236+0.126 \ln \operatorname{Re}_{d c} \\
& c_{3}=-0.361-\frac{0.042 N_{r}}{\ln \operatorname{Re}_{d c}}+0.158 \ln \left[N_{r}\left(\frac{p_{f}}{d_{c}}\right)^{0.41}\right] \\
& c_{4}=-1.224-\frac{0.076\left(X_{l} / D_{h}\right)^{1.42}}{\ln \operatorname{Re}_{d c}} \\
& c_{5}=-0.083+\frac{0.058 N_{r}}{\ln \operatorname{Re}_{d c}} \\
& c_{6}=-5.735+1.21 \ln \frac{\operatorname{Re}_{d c}}{N_{r}}
\end{aligned}
$$

Outside heat transfer coefficient is given as

$$
h=j G_{\max } C_{p}\left(P_{r}\right)^{-2 / 3}
$$




\subsection{Fin efficiency}

To determine the overall surface efficiency for a finned tube heat exchanger, it is necessary to determine the efficiency of the fins.Fin efficiency is calculated using Schmidt (1945) correlation the empirical relation for the equivalent radius is given by

$$
\frac{R_{e}}{r}=1.27 \psi(\beta-0.3)^{1 / 2}
$$

The coefficients $\psi$ and $\beta$ are defined as

$$
\psi=\frac{X_{t}}{2 r} \text { and } \beta=\frac{1}{X_{t}}\left(X_{l}^{2}+\frac{X_{t}{ }^{2}}{4}\right)^{1 / 2}
$$

The fin efficiency can be expressed as

$$
\eta_{f}=\frac{\tanh (m \cdot l)}{m \cdot l}
$$

where, $l=R_{e}-r$

The total surface efficiency of the fin, $\eta_{o}$ is therefore expressed as

$$
\eta_{o}=1-\frac{A_{f}}{A_{t}}\left(1-\eta_{f}\right)
$$

\subsection{Refrigerant side heat transfer}

The refrigerant heat transfer is calculated using the Dittu-Boelter correlation which is valid for fully developed flow in circular tubes with moderate temperature variations (Incropera\& DeWitt, 1996).

$$
N u_{D}=0.023 \operatorname{Re}_{D}^{0.8} \operatorname{Pr}^{0.3}
$$

The two-phase flow heat transfer model developed by Shah is a simple correlation which is given as

$$
\bar{h}_{T P}=\bar{h}_{L}\left[(1-x)^{0.8}+\frac{3.8 x^{0.76}(1-x)^{0.04}}{p_{r}^{0.38}}\right]
$$

For complete condensation, the mean two-phase heat transfer coefficient reduces to the following expression,

$$
\bar{h}_{T P M}=\bar{h}_{L}\left(0.55+\frac{2.09}{p_{r}^{0.38}}\right)
$$

\section{Optimization}

Optimization is done by varying the different geometric parameter like outer diameter of the condenser tube, fin per inch and number of rows of condenser and its effect on the coefficient of performance is graphically plotted below by keeping frontal area of condenser constant.

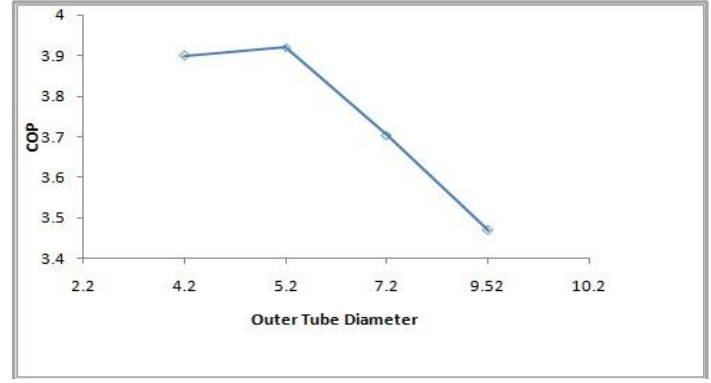

Fig.1 Effect of outer diameter on COP

After optimization it is observed that at $5.2 \mathrm{~mm}$ outer diameter, 12 fins per inch and 2 rows condense gives better performance.

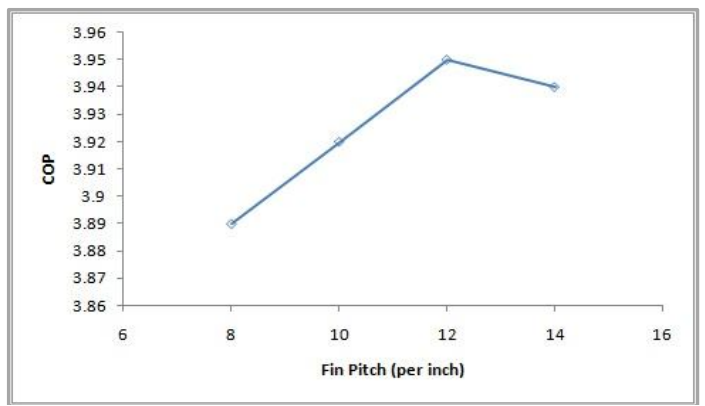

Fig.2 Effect of fin pitch on COP

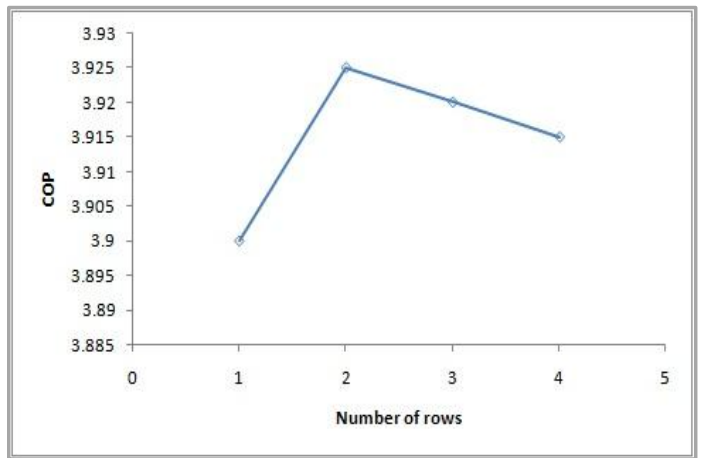

Fig.3 Effect of Number of rows on COP

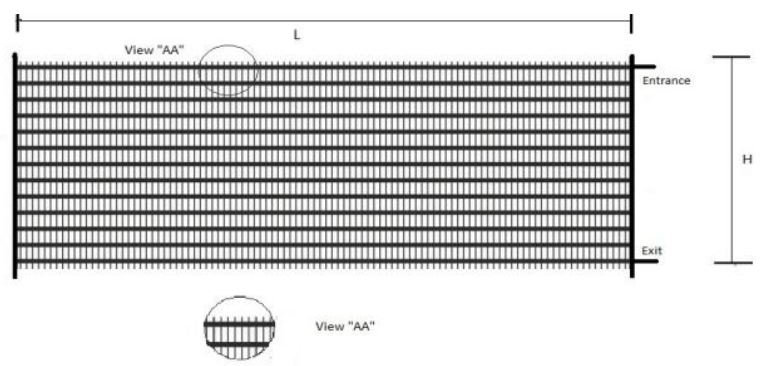

Fig.4 Condenser

Table 4 Specification of Condenser

\begin{tabular}{|c|c|}
\hline Tube material & Aluminum \\
\hline Outer diameter of the tubes $D_{o}$ & $5.2 \mathrm{~mm}$ \\
\hline Number of tubes $N_{t}$ & 13 \\
\hline Number of rows (z) & 2 \\
\hline Fin pitch & $1 \mathrm{~mm}$ \\
\hline Thickness of the fins $\delta$ & $0.20 \mathrm{~mm}$ \\
\hline
\end{tabular}




\section{Experimental Setup}

In experiment the performance analysis is done by varying the Air velocity (m/s) by condenser fan. At differentAir velocity different temperature are calculate like temperature at compressor inlet and outlet,temperature after condenser and also temperature after evaporator.

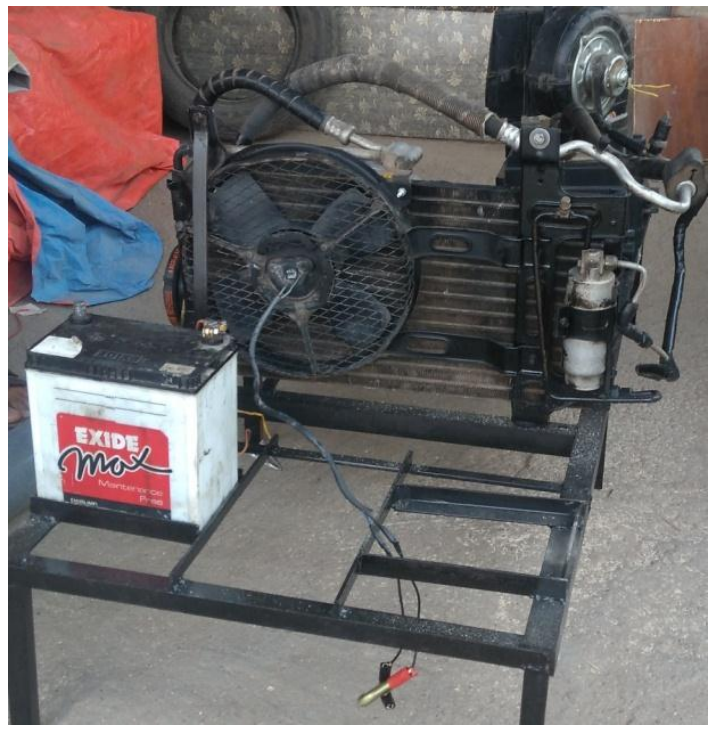

Fig.5 Experimental setup

\section{Result and discussion}

From above study of design optimization of condenser it concluded that at diameter $5.2 \mathrm{~mm} 2$ rows and 12 fins per inch gives better result as compared to other varying geometric parameter by keeping frontal area of condenser constant. As the diameter, Number of rows and fin per inch increases there will be drop in COP.

In experimentally analysis COP and Refrigerating effect is calculated and it is observed that as the air velocity increases the COP is going to increases at Air velocity $7 \mathrm{~m} \backslash \mathrm{s}$ gives better coefficient of performance.

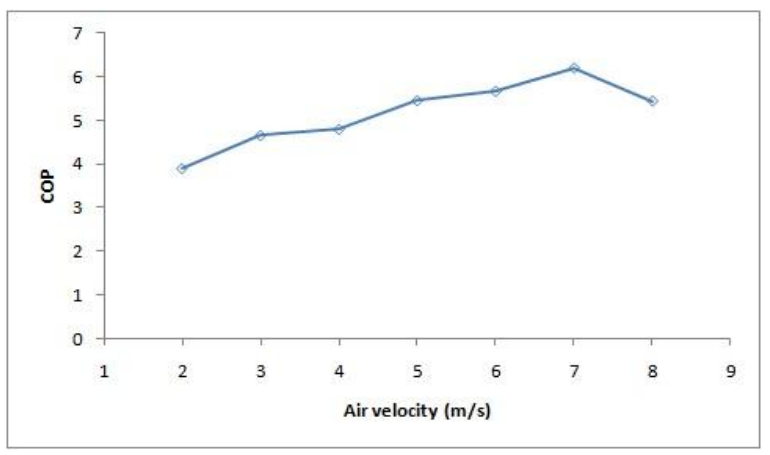

Fig.6 COP vs. Air velocity

Also in Experimental analysis it is observed that as Air velocity increased it directly affect the refrigerant effect. As Air velocity increases Refrigerant effect also increases at Air velocity $6 \mathrm{~m} \backslash \mathrm{s}$ gives optimum refrigerating effect.

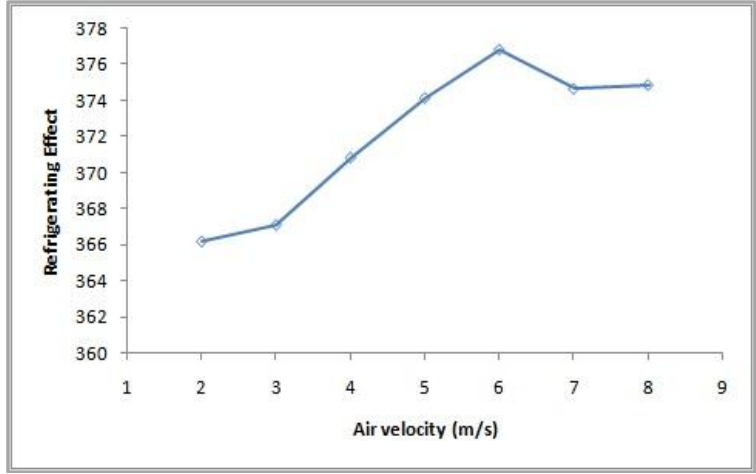

Fig.7 R.E vs. Air velocity

\section{Conclusions}

In optimization it is concluded that by keeping the frontal area of condenser constant and varying geometrical parameter it is observed that as the outer diameter increases the COP of the system is decreases. Also from varying the number of rows and fin per inch it is concluded that at 12 fins per inch gives better performance and at 2 number of rows gives better performance.Experimentally analysis it is concluded air flow velocity (condenser fan speed) also contribute effect part in improving the performance of system from experiment it is that observed that COP and R.E of the system increases with Air velocity increased.

\section{References}

R.K. Shah (2006), Automotive air-conditioning systems historical developments, the state of technology and future trends, Proceedings of the 3rd BSME-ASME International Conference on Thermal Engineering, Dhaka Bangladesh

S. Sharmas Vali and M. L. S Deva Kumar (2011) ,Experimental Analysis of the Effect of Varying the Condenser FINS Spacing on Vapor Compression Refrigeration System Performance,International Journal of Mechanical Engineering Volume 4

J.B. Copetti and M.H. Macagnan(2009), design and optimization of minichannel parallel flow condensers, Seventh International Conference on Enhanced, Compact and Ultra-Compact Heat Exchangers: From Microscale Phenomena to Industrial Applications.

Vivek Sahu and Pooja Tiwari (2013), Experimental Investigation of the Refrigerator Condenser By Varying the Fins Spacing of the Condenser, ISSN (Print): 2321-5747, Volume-1, Issue-1, 2013.

John C. Hipchen, (2012), Simulation-Based Comparison of Optimized AC Coils Using Small Diameter Copper and Aluminum Micro-Channel Tubes,International Refrigeration and Air Conditioning Conference. Paper 1305.

Jignesh K. Vaghela(2015), Theoretical And Experimental Evaluation of Automobile Air-Conditioning System UsingR134a,International Journal of Science, Technology \& ManagementVolume No 04

Patil D.P. Bhangale J.H. and Deshmukh K.S.(2014),Review paper on refrigeration system using microchannel condenser ,International Research Journal of Mechanical Engineering (IRJME) Volume 1

Mehendale Sunil, Aute Vikrant; and Radermacher Reinhard (2014) ,Principles of Refrigerant Circuit Optimization in Single Row Microchannel Condensers, International Refrigeration and Air Conditioning Conference.

Del Col, Davide; Stefano, Bortolinand Matteo, Bortolato (2012), Condensation Heat Transfer and Pressure Drop with Propane in a Minichannel, International Refrigeration and Air Conditioning Conference.

Yoo, Seong Yeon and Lee, Dae Woong (2004), An Experimental Study on Performance of Automotive Condenser and Evaporator, International Refrigeration and Air Conditioning Conference.

Mao-Gang He, Xin-Zhou Song, Huan Liu, Ying Zhang(2014),Application of natural refrigerant propane and propane/isobutene in large capacity chest freezer, Applied Thermal Engineering 70 732e736.

M. M. Rahman, H. Y. Rahman, (2012) , Hydrocarbon as refrigerant for domestic air conditioner: a comparative study between R22 and R290, Elixir Thermal Engg. 53 11976-11979.

M. Mohanraj, S. Jayaraj, C. Muraleedharan (2009), Environment friendly alternatives to halogenated refrigerants-A review, International Journal of Greenhouse Gas Control 3 108-119. 